\title{
Prediction of late recurrence after radiofrequency ablation of HBV- related hepatocellular carcinoma with the age-male-albumin- bilirubin-platelets (aMAP) risk score: a multicenter study
}

\author{
Yujing Xin ${ }^{1}$, Xinyuan Zhang ${ }^{1}$, Yi Yang ${ }^{1}$, Yi Chen ${ }^{1,2}$, Yanan Wang ${ }^{1}$, Xiang Zhou ${ }^{1}$, Xiao Li $^{1}$ \\ ${ }^{1}$ Department of Interventional Therapy, National Cancer Center/National Clinical Research Center for Cancer/Cancer Hospital, Chinese Academy \\ of Medical Sciences and Peking Union Medical College, Beijing, China; ${ }^{2}$ Department of Interventional Radiology, First Hospital of Shanxi Medical \\ University, Taiyuan, China \\ Contributions: (I) Conception and design: Y Xin; (II) Administrative support: Y Xin, Y Yang, X Zhang; (III) Provision of study materials or patients: Y \\ Xin, Y Yang; (IV) Collection and assembly of data: Y Xin, Yi Chen; (V) Data analysis and interpretation: Y Xin, X Zhang; (VI) Manuscript writing: \\ All authors; (VII) Final approval of manuscript: All authors. \\ Correspondence to: Xiang Zhou. Department of Interventional Therapy, National Cancer Center/National Clinical Research Center for Cancer/ \\ Cancer Hospital, Chinese Academy of Medical Sciences and Peking Union Medical College, Beijing 100021, China. Email: zhouxiangncc@yeah.net.
}

Background: Long-term survivals of patients with HBV-related hepatocellular carcinoma are limited by the high incidence of tumor recurrence after radiofrequency ablation (RFA), identification of the risk factors and understanding the patterns of recurrence can help to improve the comprehensive management of patients after RFA. Therefore, the purpose of the study is to explore the prognostic value of the age-malealbumin-bilirubin-platelets (aMAP) score in patients with early-stage HBV-related hepatocellular carcinoma (HCC) receiving RFA; investigate the risk factors and patterns of late recurrence (LR); and develop a nomogram to predict recurrence-free survival (RFS).

Methods: A retrospective review of HBV-related HCC patients who underwent primary RFA from March 2012 to December 2020 was conducted. The prognostic value of the aMAP score was evaluated in a primary cohort $(n=302)$ and then further validated in an independent validation cohort $(n=143)$. The optimal threshold of aMAP scores was calculated by X-tile 3.6.1 software. A prognostic nomogram was constructed from multivariate analysis and validated in an external validation cohort.

Results: Patients with aMAP scores $\leq 63.8,63.8-67.8$, and $>67.8$ were classified into low-, medium-, and high-recurrence risk groups, respectively. The C-index to predict LR was 0.76 (95\% CI: 0.700-0.810). The high-risk group was associated with the worst RFS (HR: 5.298; 95\% CI, 2.697-10.408; $\mathrm{P}<0.001$ ) and overall survival (OS) (HR: 2.639; 95\% CI, 1.097-6.344; P=0.03) compared with medium- and low-risk groups. The aMAP score, multiple tumors and preoperative HBV DNA level were independent risk factors for LR. The proposed nomogram had excellent performance in predicting LR of HBV-related HCC [C-index: 0.82 (95\% CI: 0.772-0.870)].

Conclusions: This study demonstrated that the aMAP score can serve as an objective predictor of LR for HBV-related HCC patients after RFA. The nomogram based on preoperative HBV DNA level, aMAP score, and number of tumors can reliably help clinicians to stratify the recurrence risk of HCC patients after RFA.

Keywords: HBV-related hepatocellular carcinoma; radiofrequency ablation; recurrence; aMAP score

Submitted Aug 16, 2021. Accepted for publication Nov 03, 2021.

doi: 10.21037/jgo-21-506

View this article at: https://dx.doi.org/10.21037/jgo-21-506 


\section{Introduction}

Hepatocellular carcinoma (HCC) is one of the most common malignant tumors worldwide and is associated with an extremely poor prognosis $(1,2)$. In China, HCC is the fifth most common cancer and the second most common cause of cancer-related death, with an estimated 0.41 million new cases and 0.39 million deaths in 2020 (3). HCC caused by hepatitis B virus (HBV) infection accounts for approximately $80 \%$ of all liver cancers in China $(4,5)$. Liver transplantation and liver resection are recognized as curative treatments for early-stage HCC (EHCC) $(6,7)$ In addition, local thermal ablation has been suggested as a suitable first-line alternative treatment for small HCC (8-10). Radiofrequency ablation (RFA) is one of the most widely used thermal ablation methods for EHCC and has achieved favorable oncological outcome compared with liver resection (11-13). However, the recurrence rate after ablation is high, which limits the long-term survival of HCC patients, and studies have found higher recurrence rate and poorer long-term survival in patients with HCC after RFA, compared to patients with HCC after surgical resection (14-16). Therefore, determining the risk factors for recurrence after RFA is crucial for clinicians to develop individualized post-RFA adjuvant treatment.

Based on the patterns of recurrence as reported by previous studies, HCC recurrence occurring within two years after curative treatment such as RFA or surgery is categorized early recurrence (ER) while recurrence after two years is termed as late recurrence (LR) (17-19). ER has been shown to most likely originate from micro-metastases of the initial tumor $(19,20)$. In contrast, LR is considered to be a de novo tumor with a different clonal origin from the initial tumor $(17,21)$. Previous studies suggested that aggressive characteristics of the initial tumor, including microvascular and macrovascular invasion, multinodularity, and satellite nodules, are ER-related risk factors, while LR is considered to be closely related to hepatic function and host-related characteristics such as active hepatitis, cirrhosis, age, and gender $(21,22)$. In addition, for HBV-related HCC, viral-related factors such as high viral load were identified as independent risk factors for LR $(17,23,24)$. Previous studies have demonstrated that the recurrence patterns and survival outcomes of patients with LR are different from those of patients with ER. Compared with patients with ER, patients with LR often present with multiple lesions but have better survival outcomes $(25,26)$. Therefore, identifying reliable and accurate markers for the early detection of LR of HCC is critical. However, only few studies with limited sample size have examined LR in HCC patients $(27,28)$.

Recently, a novel HCC risk score, the age-male-ALBIplatelets (aMAP) score, was developed and validated in a global, multi-ethnic, multi-etiological, prospective cohort study of chronic hepatitis to accurately predict the risk of hepatitis-related HCC (29). Age and gender were shown to be related to HCC recurrence while platelet count, serum albumin and serum bilirubin levels were co-related with liver function in multiple studies $(17,25,30,31)$. Therefore, this scoring system accurately stratifies the risk of HCC development for patients with hepatitis and can improve early HCC detection by developing personalized monitoring strategies. Given that LR of HCC is considered as a de novo secondary primary cancer that originates from the residual diseased liver (21), we hypothesized that the aMAP score may have the potential to predict the risk of LR in patients with HBV-related HCC undergoing RFA.

LR-related factors and patterns of HCC after hepatectomy or liver transplantation have been explored in previous studies $(32,33)$. However, few reports have investigated the risk factors and patterns of LR of HCC after RFA. To the best of our knowledge, the predictive ability of aMAP score for LR in HBV-associated HCC after RFA has not been studied. Hence, this study was conducted to evaluate the ability of the aMAP score to predict LR of $\mathrm{HBV}$-associated HCC and investigate other risk factors and patterns of LR. We established a new preoperative prognostic factor-based nomogram to identify patients with high risk of recurrence and guide clinicians to formulate a reasonable treatment plan and improve patient prognosis. We present the following article in accordance with the TRIPOD reporting checklist (available at https://dx.doi. org/10.21037/jgo-21-506).

\section{Methods}

\section{Patients}

The study was conducted in accordance with the Declaration of Helsinki (as revised in 2013). The study was approved by the Institutional Review Boards of the National Cancer Center (NCC2019KZ-010) on 20 April 2019. All participants provided written informed consent for inclusion in the study. This study included primary early-stage HBV-related HCC patients (18-80 years) and underwent RFA as a curative treatment between March 2012 to December 2020 at the National Cancer Center 
of China and First Hospital of Shanxi Medical University. Patients who met the following criteria were included in our study: (I) HCC diagnosed by histopathology or noninvasive diagnostic guidelines (34); (II) performance status of 0 or 1 based on Eastern Cooperative Oncology Group score; (III) EHCC patients with chronic HBV infection (solitary tumor $\leq 3 \mathrm{~cm}$ or up to three tumors $\leq 3 \mathrm{~cm}$ ); (IV) complete tumor ablation; $(\mathrm{V})$ positive hepatitis $\mathrm{B}$ surface antigen (HBsAg) for at least 6 months. We excluded patients based on the following criteria: (I) patients who received other curative treatment including hepatic resection or hepatic transplantation; (II) patients with other types of hepatitis (e.g., alcoholic hepatitis, hepatitis A or C, nonalcoholic fatty liver disease, etc.); and (III) patients who died or developed HCC recurrence within 2 years after RFA were also excluded, as this study focused on late recurrence.

\section{Data collection}

Demographic, laboratory, and clinical data were collected. Demographic data included gender, age, performance status, presence of cirrhosis, and Child-Pugh class. Hepatitis-related variables included preoperative HBVDNA load, HBV envelope antigen, history of anti-HBV therapy, and hepatic function indices, such as albuminbilirubin (ALBI) grade and aspartic transaminase (AST), among others. Tumor-related factors included maximum tumor size, tumor number, and serum alpha-fetoprotein (AFP). Cirrhosis was defined by liver function tests, medical imaging, and etiology according to the Chinese Society of Hepatology and liver cirrhosis guidelines of EASL $(35,36)$.

\section{RFA procedure and postoperative follow-up strategy}

RFA was performed by doctors with more than 8 years of ablation experience under the guidance of computed tomography (CT) or ultrasound. Local anesthesia was administered before RFA. All tumor lesions were completely ablated (the safe margin of ablation was at least $5 \mathrm{~mm}$ ) $(37,38)$. Enhancement imaging such as contrast-enhanced ultrasound or multi-stage enhanced magnetic resonance imaging (MRI) was performed immediately after RFA to confirm that the focus was completely ablated. If residual tumors were found, then repeat RFA was performed until complete ablation was achieved. Follow-up examination was performed every two months in the first year after RFA, once every 3 months in the second year, and once every 6 months thereafter until the tumor relapsed or the patient died. The last follow-up date was February 28, 2021. Follow-up examination included routine radiological imaging such as ultrasound and CT and laboratory tests such as hepatic function test and serum AFP level. Recurrent tumors after RFA were treated by hepatectomy, repeated ablation, systemic therapy (i.e., sorafenib, immunotherapy), or transarterial chemoembolization on a case-by-case basis $(39,40)$.

\section{Oncological outcomes}

The primary endpoint of this study was late recurrence (LR) or recurrence-free survival (RFS), and the secondary endpoint was overall survival (OS). The period from the initial complete ablation to the first LR of HCC was defined as RFS (more than 2 years). The period from the initial ablation to the last follow-up or death was defined as OS. Tumor recurrence was suspected in patients with a serum AFP level of more than $20 \mathrm{ng} / \mathrm{mL}$ and new lesions appeared on US/CT during routine surveillance. Tumor recurrence was confirmed by dynamic CT or MR imaging. Recurrent tumors were categorized based on the location of the tumor as follows: intrahepatic recurrence and extrahepatic recurrence (15).

\section{Statistical analysis}

Continuous variables and categorical variables were evaluated by the $t$-test or Mann-Whitney U-test and by Fisher's exact test or $\chi^{2}$ test as appropriate, respectively. Univariate and multivariate Cox proportional hazards models were used to analyze factors related to OS and RFS. OS and RFS were evaluated by Kaplan-Meier curves. Preoperative aMAP scores were calculated using age, sex, bilirubin, albumin, and platelet count. The optimal threshold of aMAP scores was calculated by X-tile 3.6.1 software (41). The cutoff values for age and tumor size were calculated based on the mean, and the best threshold of other variables was the upper limit value of the hospital laboratory department. The accuracy of hazard ratio (HR) was revealed by a twotailed $95 \%$ confidence interval (CI). Statistical analysis was performed using $\mathrm{R}$ software (version 3.6.2). The nomogram was composed of significant factors $(\mathrm{P}<0.05)$ selected from multivariate RFS analysis. Internal validation was performed by 500 bootstraps resamples. The performance and predictive power of the proposed nomogram were evaluated by the time-dependent receiver operating characteristic (t-ROC) curve and the concordance index (C-index). The 
observed and predicted survivals were compared to calibrate the nomogram for 3-, 4-, and 5-year RFS.

\section{Results}

\section{Patient characteristics}

A total of 589 patients with HBV-related HCC from two Chinese hospitals were initially included in this study. Out of these patients, 144 patients were excluded based on the following reasons: ER $(n=118)$, incomplete ablation $(n=4)$, tumors beyond $3 \mathrm{~cm}(\mathrm{n}=17)$, and patients who were lost to follow up $(\mathrm{n}=5)$. For validation purposes, 302 patients from the China National Cancer Center were included in the training cohort, and 143 patients from the First Hospital of Shanxi Medical University were included in the independent external validation cohort The baseline characteristics of patients in the validation cohort were shown in Table S1. There was no significant difference in baseline characteristics between the training cohort and the validation cohort.

Table 1 showed the baseline characteristics of patients between the patients with LR and without LR in the training cohort. The median patient age was 57.5 years old, and the median follow-up time was 48.1 months. The training cohort included 75 patients with LR and 227 patients without LR. There were significant differences in aMAP score, gender, tumor number, and preoperative $\mathrm{HBV}$ DNA level between the two groups $(\mathrm{P}<0.001)$. Among the 75 patients with LR, 71 (94.7\%) were male, 44 (58.7\%) patients had multiple tumors, and $65(86.7 \%)$ patients had liver cirrhosis. We performed Kaplan-Meier analysis and found that the OS of patients with LR was significantly shorter than patients without LR $(\mathrm{P}=0.003)$ (Figure 1).

\section{Clinical risk factors for RFS and OS}

Univariate and multivariate survival analyses showed that aMAP score (HR: 5.298, 95\% CI: 2.697-10.408; P<0.001), multiple tumors (HR: 2.549, 95\% CI: 1.561-4.161; $\mathrm{P}<0.001$ ), and preoperative HBV DNA (HR: 1.917, 95\% CI: 1.084-3.392; $\mathrm{P}=0.03)$ were independent factors affecting LR (Table 2). In addition, aMAP score (HR: 2.639, 95\% CI: 1.097-6.344; $\mathrm{P}=0.03$ ), tumor number (HR: 5.368, 95\% CI: 1.986-14.507; $\mathrm{P}<0.001$ ), and cirrhosis (HR: $3.429,95 \% \mathrm{CI}$ : 1.338-8.789; $\mathrm{P}=0.01)$ were independent factors associated with OS (Table 3).

\section{Risk stratification of LR of HCC after RFA based on aMAP score}

The two best cut-off values of aMAP score (63.8 and 67.8) were obtained using $\mathrm{X}$ software and used to divide the patients into high-, medium- and low-risk groups. The aMAP score was an independent hazard factor for LR. Kaplan-Meier survival analysis curve revealed significant differences in RFS among the high-, medium- and low-risk groups (Figure $2 A$ ). The high-risk aMAP score group was associated with worse RFS (HR, 5.298; 95\% CI, 2.697$10.408 ; \mathrm{P}<0.001)$ and $\mathrm{OS}(\mathrm{HR}, 2.639 ; 95 \% \mathrm{CI}, 1.097-$ 6.344; $\mathrm{P}=0.03)$. The 5 -year cumulative recurrence rates of the high-, medium-, and low-risk groups were $85.1 \%$, $41.5 \%$ and $19.7 \%$, respectively (all $\mathrm{P}<0.05$ ). The 5 -year cumulative OS rates of the high-, medium-, and lowrisk groups were $58.6 \%, 74.0 \%$, and $97.8 \%$, respectively (Figure 2B). We achieved a C-index of 0.76 (95\% CI: 0.700-0.810) using the aMAP score to predict LR, and the t-AUCs of the aMAP score of 3-, 4-, and 5-year RFS were $0.73,0.76$, and 0.72 , respectively. The independent external validation cohort further confirmed the prediction value of the aMAP score and achieved a C-index of 0.77 (95\% CI: $0.700-0.842$ ) to predict LR; the t-AUCs of the aMAP score of 3-, 4-, and 5-year RFS were $0.73,0.77$, and 0.77 , respectively.

\section{Development, verification, and calibration of the nomogram}

A nomogram including aMAP score, tumor number, and preoperative HBV DNA level was proposed to predict the 3-, 4-, and 5-year RFS based on the results of multivariate Cox proportional hazards model (Figure 3). The C-index of the nomogram was 0.82 (95\% CI: $0.772-0.870)$. The t-AUCs of the nomogram of 3-, 4-, and 5-year RFS were $0.82,0.83$, and 0.77 , respectively. We found that the nomogram performed better than the traditional reference markers in predicting LR (Figure 4A).

The prognostic performance of the nomogram was subsequently tested in the independent external validation cohort (Figure 4B). The C-index was 0.82 (95\% CI: $0.750-0.884)$, and the t-AUCs of the nomogram of 3-, 4-, and 5 -year RFS were $0.81,0.84$, and 0.81 , respectively. The calibration curves showed that the proposed nomogram fitted well in the two independent cohorts (Figure 5). 
Table 1 Baseline demographics of patients in the training cohort

\begin{tabular}{|c|c|c|c|c|}
\hline Characteristics & Total patients & No late recurrence, $\mathrm{n}(\%)$ & Late recurrence, n (\%) & $P$ value \\
\hline aMAP score & & & & $<0.001$ \\
\hline$\leq 63.8$ & 204 & $181(79.7)$ & $23(30.7)$ & \\
\hline $63.9-67.8$ & 61 & $43(18.9)$ & $18(24.0)$ & \\
\hline Age (years) & & & & 0.091 \\
\hline$\leq 60$ & 195 & $140(61.7)$ & 55 (73.3) & \\
\hline$>60$ & 107 & 87 (38.3) & $20(26.7)$ & \\
\hline Gender & & & & $<0.001$ \\
\hline Max size $(\mathrm{cm})$ & & & & 1.000 \\
\hline$\leq 2.2$ & 171 & $98(43.2)$ & $33(44.0)$ & \\
\hline$>2.2$ & & $129(56.8)$ & $42(56.0)$ & \\
\hline Tumor number & & & & $<0.001$ \\
\hline One & 224 & $193(85.0)$ & $31(41.3)$ & \\
\hline Multiple & 78 & $34(15.0)$ & $44(58.7)$ & \\
\hline Antiviral therapy & & & & 0.084 \\
\hline No & 149 & $105(46.3)$ & $44(58.7)$ & \\
\hline Yes & 44 & $12(5.3)$ & $32(42.7)$ & \\
\hline No & 258 & $215(94.7)$ & $43(57.3)$ & \\
\hline $\mathrm{HBsAg}(\mathrm{U} / \mathrm{mL})$ & & & & 0.194 \\
\hline$\leq 250$ & 194 & $151(66.5)$ & $43(57.3)$ & \\
\hline$>250$ & 108 & $76(33.5)$ & $32(42.7)$ & \\
\hline Cirrhosis & & & & 0.389 \\
\hline No & 83 & $59(26.0)$ & 24 (32) & \\
\hline Yes & 219 & $168(74.0)$ & $51(68.0)$ & \\
\hline \multicolumn{5}{|l|}{ Child-Pugh class } \\
\hline A & 293 & $219(96.5)$ & $74(98.7)$ & 0.565 \\
\hline B & 9 & $8(3.5)$ & $1(1.3)$ & \\
\hline
\end{tabular}

Table 1 (continued) 
Table 1 (continued)

\begin{tabular}{|c|c|c|c|c|}
\hline Characteristics & Total patients & No late recurrence, $\mathrm{n}(\%)$ & Late recurrence, n (\%) & $P$ value \\
\hline 1 & 164 & $122(53.7)$ & $42(56.0)$ & \\
\hline 2 & 138 & $105(46.3)$ & $33(44.0)$ & \\
\hline AFP $(n g / m L)$ & & & & 0.362 \\
\hline$>400$ & 35 & $29(12.8)$ & $6(8.0)$ & \\
\hline AST (U/L) & & & & 0.742 \\
\hline$\leq 40$ & 252 & $188(82.8)$ & $64(85.3)$ & \\
\hline$>40$ & 50 & $39(17.2)$ & $11(14.7)$ & \\
\hline$>40$ & 237 & $179(78.9)$ & $58(77.3)$ & \\
\hline $\operatorname{PLT}\left(10^{9} / \mathrm{L}\right)$ & & & & 0.112 \\
\hline$\leq 100$ & 104 & $72(31.7)$ & $32(42.7)$ & \\
\hline$>100$ & 198 & $155(68.3)$ & $43(57.3)$ & \\
\hline TBIL $(\mu \mathrm{mol} / \mathrm{L})$ & & & & 0.295 \\
\hline$\leq 17.1$ & 206 & $159(70.0)$ & $47(62.7)$ & \\
\hline$>17.1$ & 96 & $68(30.0)$ & $28(37.3)$ & \\
\hline$\gamma-\mathrm{GT}(\mathrm{U} / \mathrm{L})$ & & & & 0.279 \\
\hline
\end{tabular}

aMAP, age-male-albumin-bilirubin-platelet; ALBI, albumin-bilirubin; AFP, alpha-fetoprotein; AST, aspartic transaminase; ALT, alanine transaminase; PLT, platelet; TBIL, total bilirubin; $\gamma$-GT, gamma-glutamyltransferase.

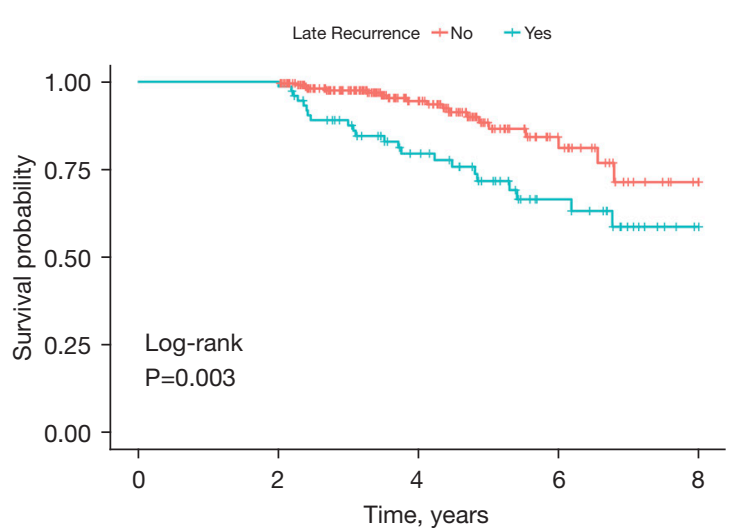

Figure 1 Kaplan-Meier curve for OS of HCC patients with and without late recurrence after RFA. OS, overall survival; HCC, hepatocellular carcinoma; RFA, radiofrequency ablation.

\section{Patterns and treatment of LR after RFA}

Among the 75 patients who developed late recurrence, $68(90.7 \%)$ were first diagnosed to have intrahepatic recurrence only and $7(9.3 \%)$ to have intrahepatic and extrahepatic recurrence. There were no patients who had extrahepatic metastases only without intrahepatic recurrence. Multiple tumors were present in $56 \%$ of recurrent cases. LR was treated as follows: hepatectomy $(50.7 \%)$, repeat ablation $(40.0 \%)$, transarterial chemoembolization $(5.3 \%)$, systemic therapy $(2.7 \%)$, and best supportive care $(1.3 \%)$.

\section{Discussion}

This multicenter retrospective study found that the aMAP 
Table 2 Cox regression analyses of risk factors for late recurrence

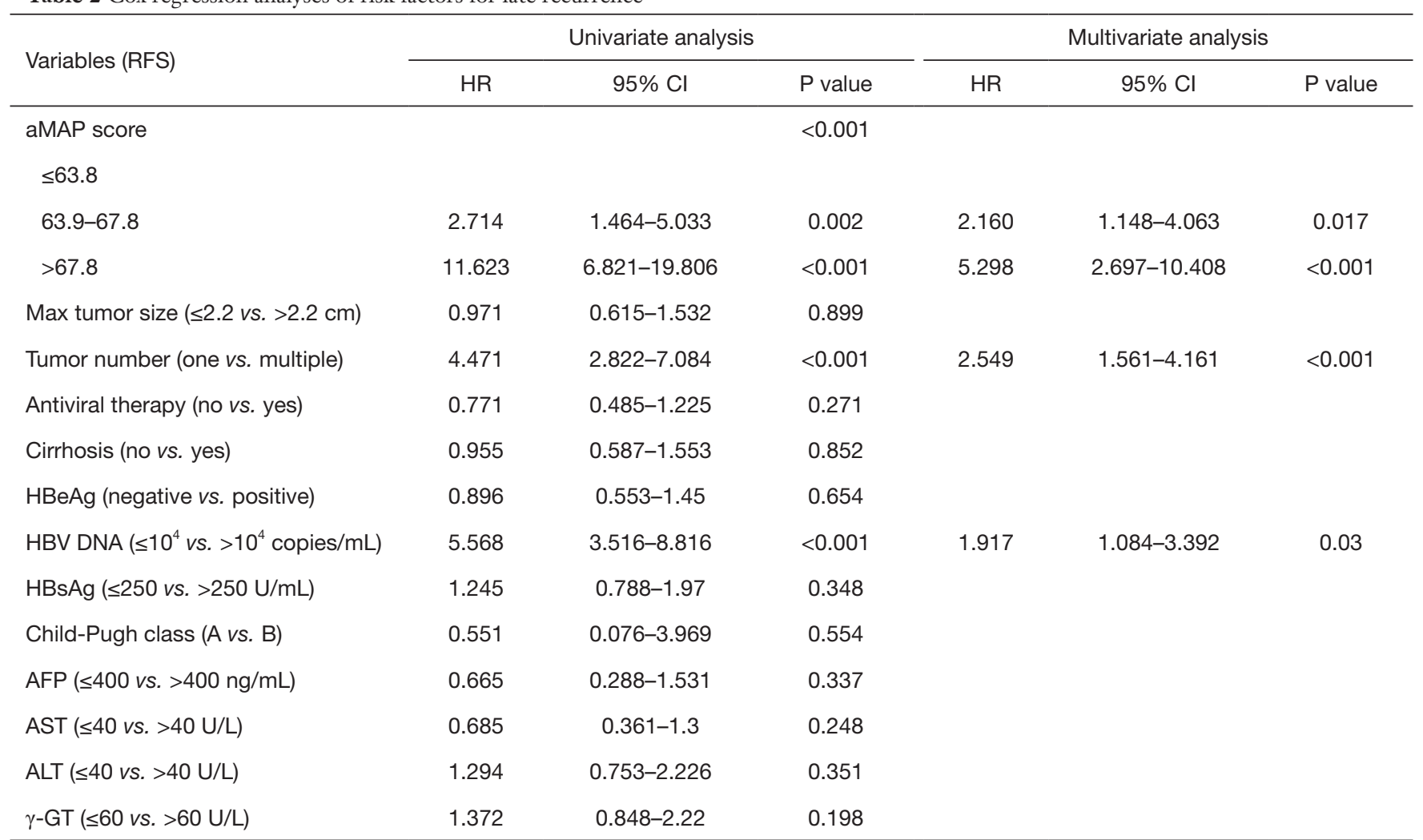

aMAP, age-male-albumin-bilirubin-platelet; ALBI, albumin-bilirubin; AFP, alpha-fetoprotein; AST, aspartic transaminase; ALT, alanine transaminase; PLT, platelet; TBIL, total bilirubin; $\gamma$-GT, gamma-glutamyltransferase.

score appropriately discriminated among low, medium, and high-risk groups for LR in HBV-related HCC after RFA. In addition, multiple tumors, preoperative HBV DNA level, and aMAP score were identified as independent predictive factors for LR, and the proposed nomogram had excellent performance in assessing LR of HBV-related HCC. These results indicate that the nomogram can be used by clinicians to develop individualized follow-up strategies and adjuvant treatment after RFA.

The high recurrence rate of HCC after RFA significantly affects the long-term survival of HCC patients in clinical practice. In this study, we first evaluated the predictive value of the aMAP score for LR after RFA and then verified it in an independent cohort. The high-risk group with an aMAP score $>67.8$ had the worst RFS and OS compared with medium-risk and low-risk groups. Hence, the highrisk group of patients should receive close surveillance after RFA for timely detection of LR. The variables included in the aMAP score, namely, age, male, ALBI, and platelet count, have been found to be associated with LR of HCC in previous studies $(42,43)$. ALBI reflects hepatic function, and platelet count represents the degree of hepatic fibrosis (44). Most previous studies also confirmed that male gender is associated with higher recurrence rates of HCC $(45,46)$. Notably, these variables can be easily obtained to calculate aMAP score in clinical practice and are not affected by virus-related factors such as viral load and antiviral drugs. Therefore, the aMAP score is suitable for predicting LR in patients with HBV-related HCC, regardless of the history of antiviral therapy.

Understanding the recurrence patterns of LR of HBVrelated HCC is vital for clinicians to design appropriate follow-up strategies and guide treatment. The main pattern of LR in this study was the presence of multiple intrahepatic metastases. This finding supports the hypothesis that LR develops in the background of hepatitis or cirrhosis and it that LR is a de novo second primary cancer with a different clonal origin than that of initial tumor $(15,21,26)$. A high viral load has been reported to be associated with LR of HCC, and antiviral therapy 
Table 3 Cox regression analyses of risk factors for OS

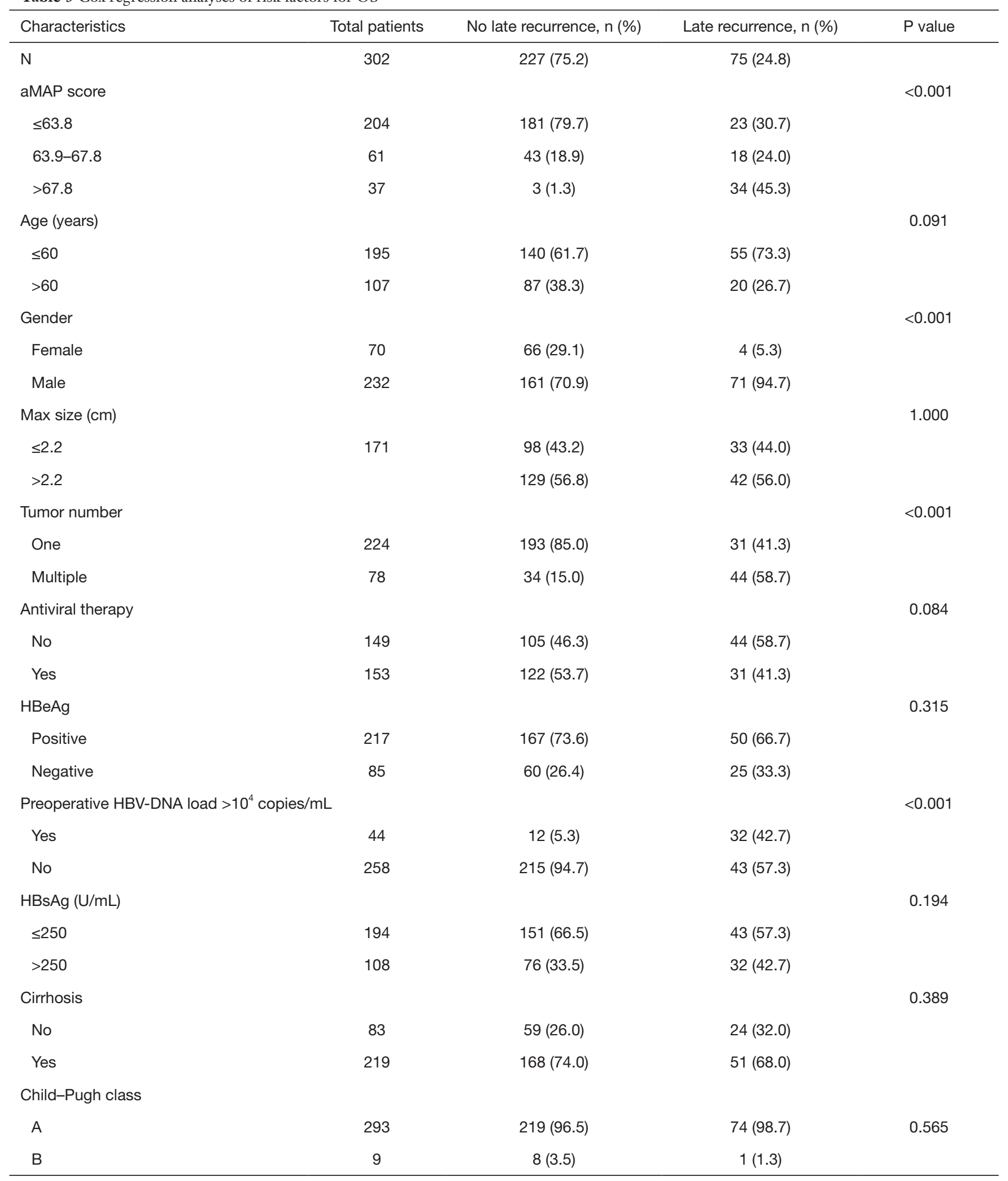

Table 3 (continued) 
Table 3 (continued)

\begin{tabular}{|c|c|c|c|c|}
\hline Characteristics & Total patients & No late recurrence, $\mathrm{n}(\%)$ & Late recurrence, $\mathrm{n}(\%)$ & $P$ value \\
\hline 1 & 164 & $122(53.7)$ & $42(56.0)$ & \\
\hline 2 & 138 & $105(46.3)$ & $33(44.0)$ & \\
\hline AFP (ng/mL) & & & & 0.362 \\
\hline$>400$ & 35 & $29(12.8)$ & $6(8.0)$ & \\
\hline AST (U/L) & & & & 0.742 \\
\hline$\leq 40$ & 252 & $188(82.8)$ & $64(85.3)$ & \\
\hline$>40$ & 50 & $39(17.2)$ & $11(14.7)$ & \\
\hline$>40$ & 237 & $179(78.9)$ & $58(77.3)$ & \\
\hline PLT $\left(10^{9} / \mathrm{L}\right)$ & & & & 0.112 \\
\hline$\leq 100$ & 104 & $72(31.7)$ & $32(42.7)$ & \\
\hline$>100$ & 198 & $155(68.3)$ & $43(57.3)$ & \\
\hline TBIL $(\mu \mathrm{mol} / \mathrm{L})$ & & & & 0.295 \\
\hline$\leq 17.1$ & 206 & $159(70.0)$ & $47(62.7)$ & \\
\hline$>17.1$ & 96 & $68(30.0)$ & $28(37.3)$ & \\
\hline$\gamma-\mathrm{GT}(\mathrm{U} / \mathrm{L})$ & & & & 0.279 \\
\hline
\end{tabular}

aMAP, age-male-albumin-bilirubin-platelet; ALBI, albumin-bilirubin; AFP, alpha-fetoprotein; AST, aspartic transaminase; ALT, alanine transaminase; PLT, platelet; TBIL, total bilirubin; $\gamma$-GT, gamma-glutamyltransferase.
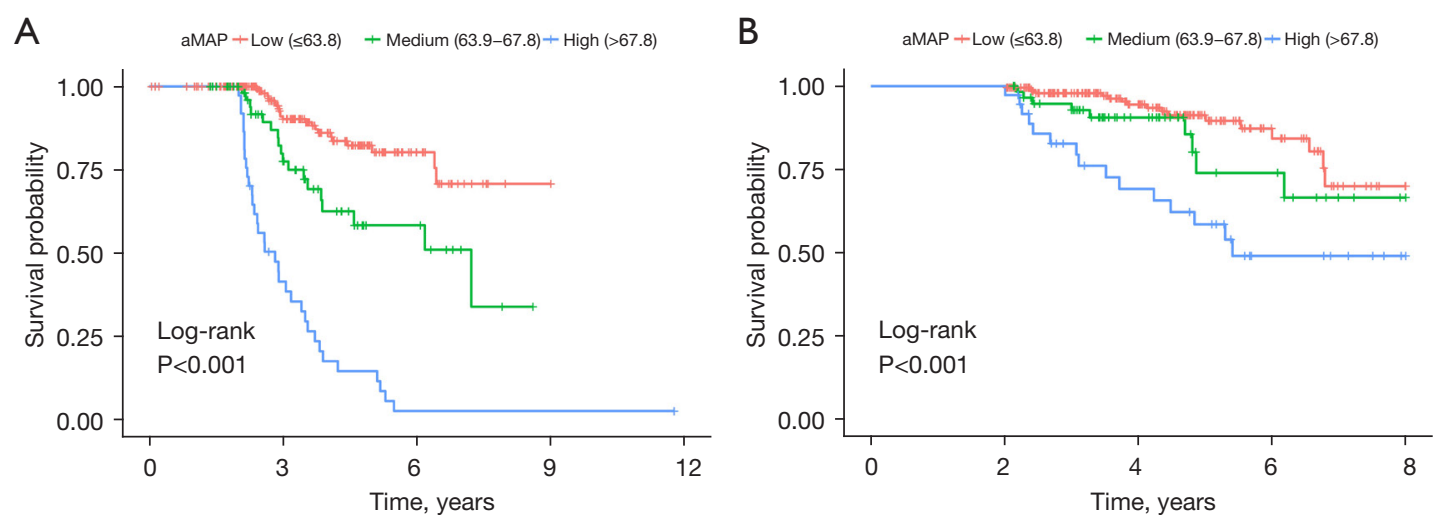

Figure 2 Kaplan-Meier curves for RFS (A) and OS (B) of patients in low-risk, medium-risk and high-risk groups. RFS, recurrence-free survival; OS, overall survival. 


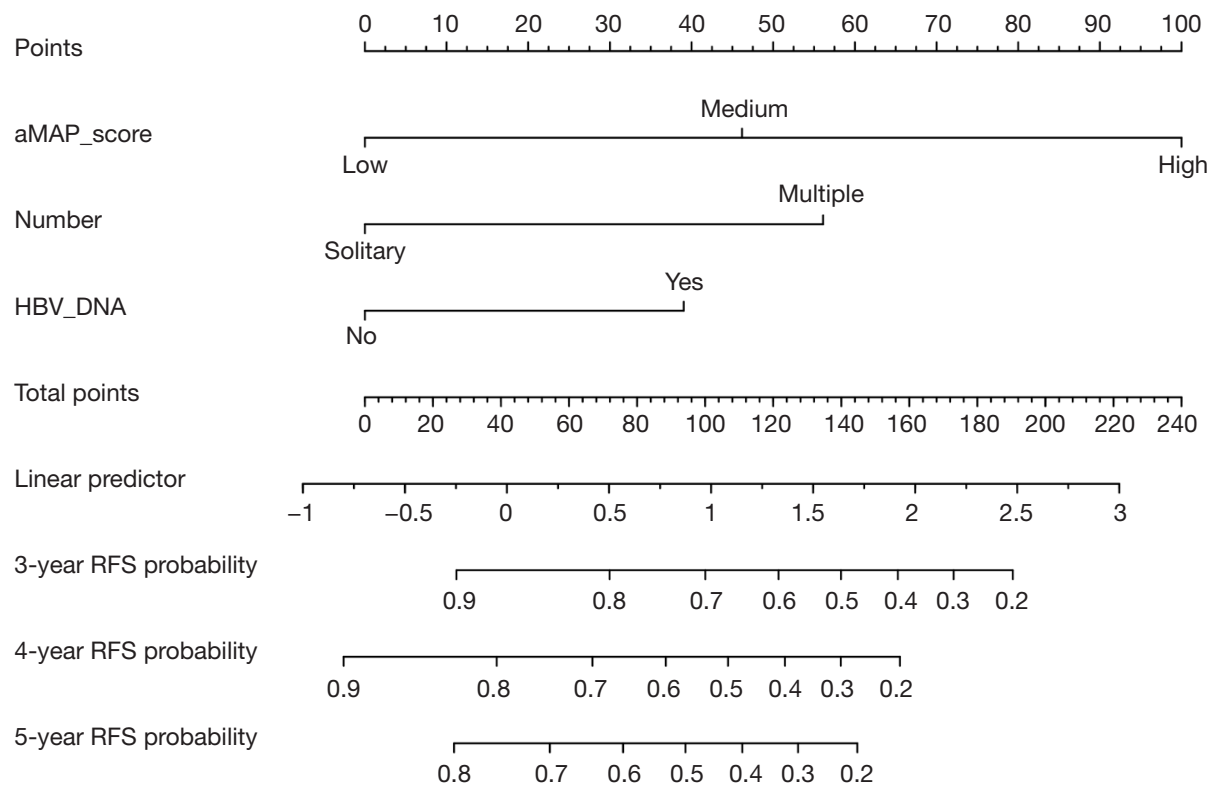

Figure 3 The nomogram for predicting the RFS after RFA. RFS, recurrence-free survival; RFA, radiofrequency ablation.
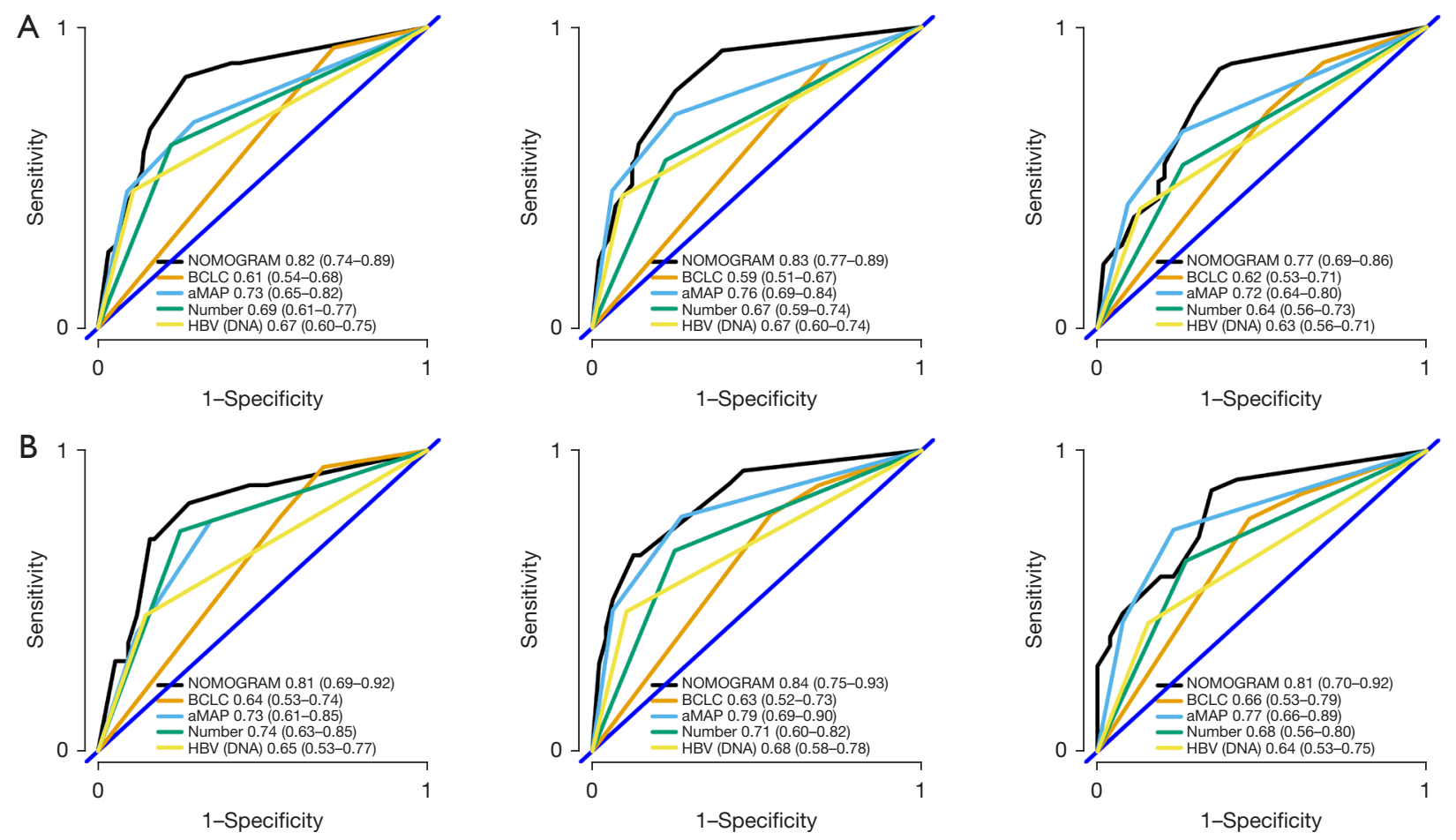

Figure 4 The t-ROC of the nomogram predicting the 3-, 4- and 5-year RFS in the training cohort (A) and the validation cohort (B). t-ROC, time-dependent receiver operating characteristic; RFS, recurrence-free survival. 

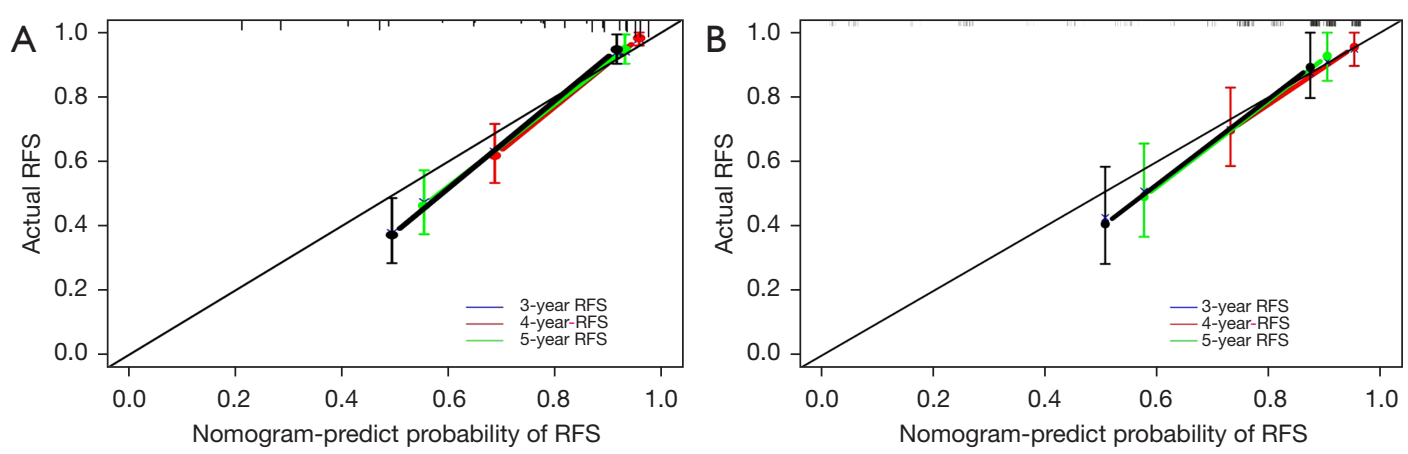

Figure 5 The calibration curves predicting RFS after RFA in the training cohort (A) and the validation cohort (B). RFS, recurrence-free survival; RFA, radiofrequency ablation.

has been proven to reduce the recurrence rate (47). This study also found that elevated serum HBV DNA level $\left(>10^{4}\right.$ copies $\left./ \mathrm{mL}\right)$ is a strong risk predictor of HCC late recurrence independent of $\mathrm{HBeAg}$ and $\mathrm{HBsAg}$. An active HBV-DNA replication in HCC may initiate hepatocarcinogenesis via both direct and indirect carcinogenic mechanisms after RFA. Therefore, routine antiviral and anti-inflammatory treatment is the key to decrease the LR rate in patients with HBV-related HCC. The nomogram proposed in this study integrated the aMAP score, virus-related factors, and tumor load and accurately and reliably identified patients at a high risk for LR.

Despite promising results, this study has several limitations. First, this retrospective study had a small sample size and inherent limitations. Second, the diversity in the treatment of LR after RFA directly affected the OS of the study patients; therefore, we took RFS as the primary endpoint instead of OS in this study. Third, this study only included patients with HCC caused by HBV infection and we did not include patients with HCC caused by other etiologies. Future prospective multicenter studies with larger sample sizes and HCC patients with other etiologies are required to validate the findings of this study. Finally, this study did not analyze the survival outcomes of different treatment methods for LR.

In summary, our results demonstrated that the aMAP score may serve as a potential and objective predictor of LR for HBV-related HCC patients after RFA. The nomogram based on preoperative HBV DNA level, aMAP score, and multiple tumors may reliably help clinicians stratify the risk of LR to formulate personalized follow-up strategies and adjuvant therapy after RFA.

\section{Acknowledgments}

Funding: This work is supported by the National Natural Science Foundation of China (30970839\&31170957), and the Research Project of Shanxi Province Health Commission (2017045).

\section{Footnote}

Reporting Checklist: The authors have completed the TRIPOD reporting checklist. Available at https://dx.doi. org/10.21037/jgo-21-506

Data Sharing Statement: Available at https://dx.doi. org/10.21037/jgo-21-506

Conflicts of Interest: All authors have completed the ICMJE uniform disclosure form (available at https://dx.doi. org/10.21037/jgo-21-506). The authors have no conflicts of interest to declare.

Ethical Statement: The authors are accountable for all aspects of the work in ensuring that questions related to the accuracy or integrity of any part of the work are appropriately investigated and resolved. The study was conducted in accordance with the Declaration of Helsinki (as revised in 2013). The study was approved by the Institutional Review Boards of the National Cancer Center (NCC2019KZ-010). All participants provided written informed consent for inclusion in the study.

Open Access Statement: This is an Open Access article 
distributed in accordance with the Creative Commons Attribution-NonCommercial-NoDerivs 4.0 International License (CC BY-NC-ND 4.0), which permits the noncommercial replication and distribution of the article with the strict proviso that no changes or edits are made and the original work is properly cited (including links to both the formal publication through the relevant DOI and the license). See: https://creativecommons.org/licenses/by-nc-nd/4.0/.

\section{References}

1. Villanueva A. Hepatocellular Carcinoma. N Engl J Med 2019;380:1450-62.

2. Kulik L, El-Serag HB. Epidemiology and Management of Hepatocellular Carcinoma. Gastroenterology 2019;156:477-491.e1.

3. Cao W, Chen HD, Yu YW, et al. Changing profiles of cancer burden worldwide and in China: a secondary analysis of the global cancer statistics 2020. Chin Med J (Engl) 2021;134:783-91.

4. Tang LSY, Covert E, Wilson E, et al. Chronic Hepatitis B Infection: A Review. JAMA 2018;319:1802-13.

5. European Association for the Study of the Liver. Electronic address: easloffice@easloffice.eu; European Association for the Study of the Liver. EASL 2017 Clinical Practice Guidelines on the management of hepatitis B virus infection. J Hepatol 2017;67:370-98.

6. Heimbach JK, Kulik LM, Finn RS, et al. AASLD guidelines for the treatment of hepatocellular carcinoma. Hepatology 2018;67:358-80.

7. Lim KC, Chow PK, Allen JC, et al. Systematic review of outcomes of liver resection for early hepatocellular carcinoma within the Milan criteria. Br J Surg 2012;99:1622-9.

8. Choi D, Lim HK, Rhim H, et al. Percutaneous radiofrequency ablation for early-stage hepatocellular carcinoma as a first-line treatment: long-term results and prognostic factors in a large single-institution series. Eur Radiol 2007;17:684-92.

9. Forner A, Reig M, Bruix J. Hepatocellular carcinoma. Lancet 2018;391:1301-14.

10. Decadt B, Siriwardena AK. Radiofrequency ablation of liver tumours: systematic review. Lancet Oncol 2004;5:550-60.

11. Rajyaguru DJ, Borgert AJ, Smith AL, et al. Radiofrequency Ablation Versus Stereotactic Body Radiotherapy for Localized Hepatocellular Carcinoma in Nonsurgically Managed Patients: Analysis of the National Cancer Database. J Clin Oncol 2018;36:600-8.

12. Gupta P, Maralakunte M, Kumar-M P, et al. Overall survival and local recurrence following RFA, MWA, and cryoablation of very early and early HCC: a systematic review and Bayesian network meta-analysis. Eur Radiol 2021;31:5400-8.

13. Huang J, Yan L, Cheng Z, et al. A randomized trial comparing radiofrequency ablation and surgical resection for HCC conforming to the Milan criteria. Ann Surg 2010;252:903-12.

14. Chu HH, Kim JH, Kim PN, et al. Surgical resection versus radiofrequency ablation very early-stage HCC $(\leq 2$ cm Single HCC): A propensity score analysis. Liver Int 2019;39:2397-407.

15. Tabrizian P, Jibara G, Shrager B, et al. Recurrence of hepatocellular cancer after resection: patterns, treatments, and prognosis. Ann Surg 2015;261:947-55.

16. Lee S, Kang TW, Cha DI, et al. Radiofrequency ablation vs. surgery for perivascular hepatocellular carcinoma: Propensity score analyses of long-term outcomes. J Hepatol 2018;69:70-8.

17. Wang MD, Li C, Liang L, et al. Early and Late Recurrence of Hepatitis B Virus-Associated Hepatocellular Carcinoma. Oncologist 2020;25:e1541-51.

18. El-Domiaty N, Saliba F, Vibert E, et al. Early Versus Late Hepatocellular Carcinoma Recurrence After Transplantation: Predictive Factors, Patterns, and Longterm Outcome. Transplantation 2021;105:1778-90.

19. Xing H, Zhang WG, Cescon $M$, et al. Defining and predicting early recurrence after liver resection of hepatocellular carcinoma: a multi-institutional study. HPB (Oxford) 2020;22:677-89.

20. Zhou SL, Zhou ZJ, Hu ZQ, et al. Genomic sequencing identifies WNK2 as a driver in hepatocellular carcinoma and a risk factor for early recurrence. J Hepatol 2019;71:1152-63.

21. Xu XF, Xing H, Han J, et al. Risk Factors, Patterns, and Outcomes of Late Recurrence After Liver Resection for Hepatocellular Carcinoma: A Multicenter Study From China. JAMA Surg 2019;154:209-17.

22. Yang Y, Chen Y, Ye F, et al. Late recurrence of hepatocellular carcinoma after radiofrequency ablation: a multicenter study of risk factors, patterns, and survival. Eur Radiol 2021;31:3053-64.

23. Wu CJ, Chau GY, Lee IC, et al. Early and late recurrence of surgically resected hepatitis B virus-related hepatocellular carcinoma on nucleos(t)ide analogues therapy. J Formos Med Assoc 2021;120:1563-71.

24. Qu LS, Jin F, Huang XW, et al. High hepatitis B viral load predicts recurrence of small hepatocellular carcinoma after curative resection. J Gastrointest Surg 2010;14:1111-20.

25. Wei T, Zhang XF, Bagante F, et al. Early Versus Late 
Recurrence of Hepatocellular Carcinoma After Surgical

Resection Based on Post-recurrence Survival: an International Multi-institutional Analysis. J Gastrointest Surg 2021;25:125-33.

26. Chok KS, Chan SC, Cheung TT, et al. Late recurrence of hepatocellular carcinoma after liver transplantation. World J Surg 2011;35:2058-62.

27. Lee S, Kim SH, Hwang JA, et al. Pre-operative ADC predicts early recurrence of HCC after curative resection. Eur Radiol 2019;29:1003-12.

28. Conti F, Buonfiglioli F, Scuteri A, et al. Early occurrence and recurrence of hepatocellular carcinoma in HCVrelated cirrhosis treated with direct-acting antivirals. J Hepatol 2016;65:727-33.

29. Fan R, Papatheodoridis G, Sun J, et al. aMAP risk score predicts hepatocellular carcinoma development in patients with chronic hepatitis. J Hepatol 2020;73:1368-78.

30. Marasco G, Colecchia A, Colli A, et al. Role of liver and spleen stiffness in predicting the recurrence of hepatocellular carcinoma after resection. J Hepatol 2019;70:440-8.

31. Lin CY, Lin CC, Wang CC, et al. The ALBI Grade is a Good Predictive Model for Very Late Recurrence in Patients with Hepatocellular Carcinoma Undergoing Primary Resection. World J Surg 2020;44:247-57.

32. Xu W, Li R, Liu F. Novel Prognostic Nomograms for Predicting Early and Late Recurrence of Hepatocellular Carcinoma After Curative Hepatectomy. Cancer Manag Res 2020;12:1693-712.

33. Cheng Z, Yang P, Qu S, et al. Risk factors and management for early and late intrahepatic recurrence of solitary hepatocellular carcinoma after curative resection. HPB (Oxford) 2015;17:422-7.

34. European Association For The Study Of The Liver; European Organisation For Research And Treatment Of Cancer. EASL-EORTC clinical practice guidelines: management of hepatocellular carcinoma. J Hepatol 2012;56:908-43.

35. European Association for the Study of the Liver. Electronic address: easloffice@easloffice.eu; European Association for the Study of the Liver. EASL Clinical Practice Guidelines for the management of patients with decompensated cirrhosis. J Hepatol 2018;69:406-60.

36. Chinese Society of Hepatology, Chinese Medical Association. Chinese guidelines on the management of liver cirrhosis. Zhonghua Gan Zang Bing Za Zhi 2019;27:846-65.

37. Liao M, Zhong X, Zhang J, et al. Radiofrequency ablation using a 10-mm target margin for small hepatocellular carcinoma in patients with liver cirrhosis: A prospective randomized trial. J Surg Oncol 2017;115:971-9.

38. Lee DH, Lee JM, Lee JY, et al. Radiofrequency ablation of hepatocellular carcinoma as first-line treatment: longterm results and prognostic factors in 162 patients with cirrhosis. Radiology 2014;270:900-9.

39. Vogel A, Cervantes A, Chau I, et al. Hepatocellular carcinoma: ESMO Clinical Practice Guidelines for diagnosis, treatment and follow-up. Ann Oncol 2018;29:iv238-55.

40. Zhang T, Zhang L, Xu Y, et al. Neoadjuvant therapy and immunotherapy strategies for hepatocellular carcinoma. Am J Cancer Res 2020;10:1658-67.

41. Camp RL, Dolled-Filhart M, Rimm DL. X-tile: a new bio-informatics tool for biomarker assessment and outcome-based cut-point optimization. Clin Cancer Res 2004;10:7252-9.

42. Hiraoka A, Kumada T, Michitaka K, et al. Newly Proposed ALBI Grade and ALBI-T Score as Tools for Assessment of Hepatic Function and Prognosis in Hepatocellular Carcinoma Patients. Liver Cancer 2019;8:312-25.

43. Zuo X, Kong W, Feng L, et al. Elevated platelet distribution width predicts poor prognosis in hepatocellular carcinoma. Cancer Biomark 2019;24:307-13.

44. Pinato DJ, Sharma R, Allara E, et al. The ALBI grade provides objective hepatic reserve estimation across each BCLC stage of hepatocellular carcinoma. J Hepatol 2017;66:338-46.

45. Lee CM, Lu SN, Changchien CS, et al. Age, gender, and local geographic variations of viral etiology of hepatocellular carcinoma in a hyperendemic area for hepatitis B virus infection. Cancer 1999;86:1143-50.

46. Cullaro G, Rubin J, Mehta N, et al. Sex-based Disparities in Hepatocellular Carcinoma Recurrence After Liver Transplantation. Transplantation 2021;105:2420-6.

47. Choi J, Jo C, Lim YS. Tenofovir Versus Entecavir on Recurrence of Hepatitis B Virus-Related Hepatocellular Carcinoma After Surgical Resection. Hepatology 2021;73:661-73.

Cite this article as: Xin Y, Zhang X, Yang Y, Chen Y, Wang Y, Zhou X, Li X. Prediction of late recurrence after radiofrequency ablation of HBV-related hepatocellular carcinoma with the age-male-albumin-bilirubin-platelets (aMAP) risk score: a multicenter study. J Gastrointest Oncol 2021;12(6):2930-2942. doi: 10.21037/jgo-21-506 
Supplementary

Table S1 Patient demographics on validation group

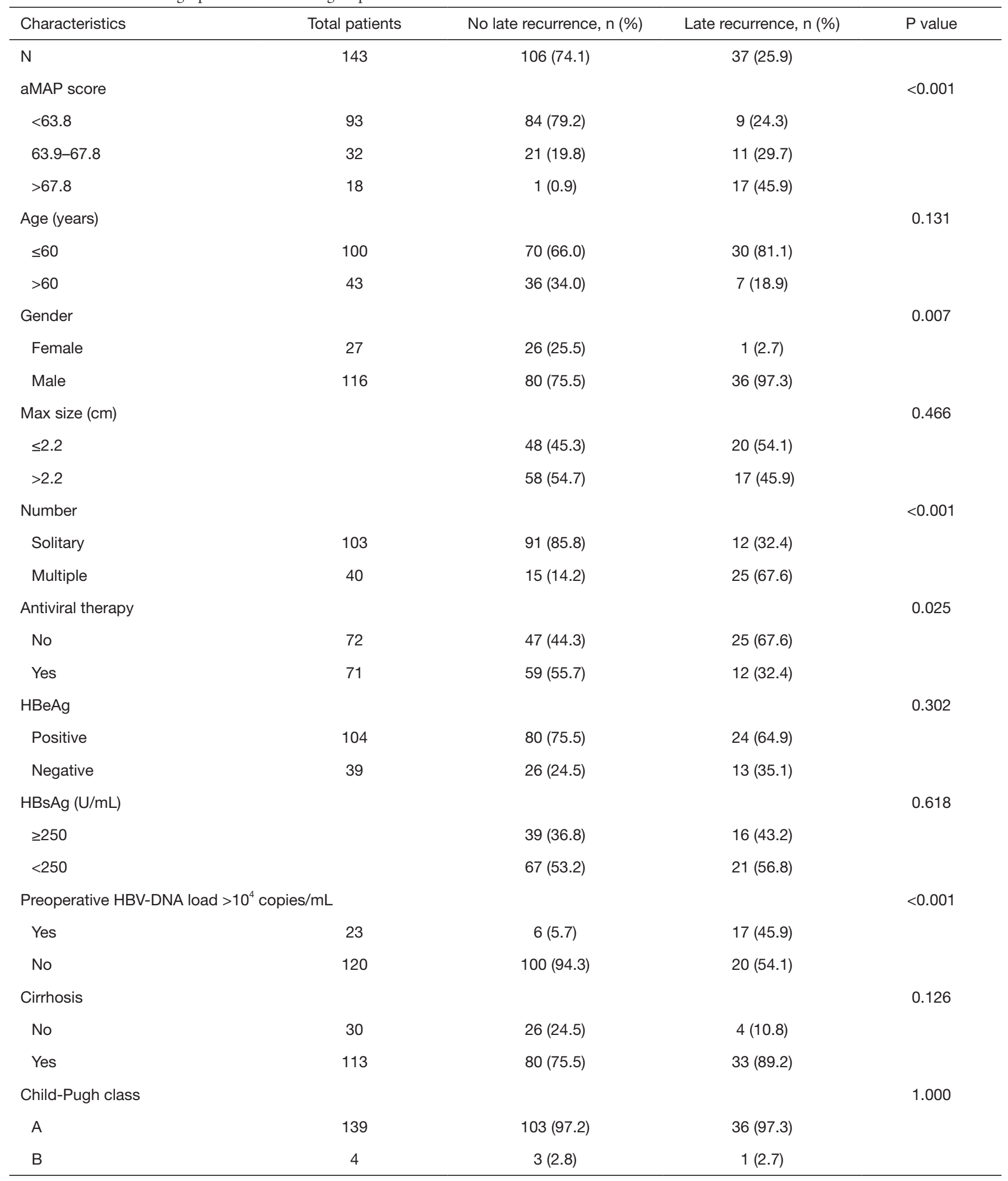

Table S1 (continued)

(C) Journal of Gastrointestinal Oncology. All rights reserved. 
Table S1 (continued)

\begin{tabular}{|c|c|c|c|c|}
\hline Characteristics & Total patients & No late recurrence, $\mathrm{n}(\%)$ & Late recurrence, n (\%) & $P$ value \\
\hline 1 & 83 & $58(54.7)$ & $25(67.6)$ & \\
\hline 2 & 60 & $48(45.3)$ & $12(32.4)$ & \\
\hline AFP (ng/mL) & & & & 0.216 \\
\hline$>400$ & 13 & $12(11.3)$ & $1(2.7)$ & \\
\hline ALB $(g / L)$ & & & & 0.093 \\
\hline$\leq 35$ & 11 & $11(10.4)$ & $0(0)$ & \\
\hline$>35$ & 132 & $95(89.6)$ & $37(100.0)$ & \\
\hline$>40$ & 24 & $19(17.9)$ & $5(13.5)$ & \\
\hline ALT (U/L) & & & & 0.721 \\
\hline$\leq 40$ & 32 & $25(23.6)$ & 7 (18.9) & \\
\hline$>40$ & 111 & $81(76.4)$ & $30(81.1)$ & \\
\hline PLT $\left(10^{9} / L\right)$ & & & & 0.271 \\
\hline$\leq 100$ & 53 & $36(34.0)$ & $17(45.9)$ & \\
\hline$>100$ & 90 & $70(66.0)$ & $20(54.1)$ & \\
\hline TBIL $(\mu \mathrm{mol} / \mathrm{L})$ & & & & 0.603 \\
\hline
\end{tabular}

aMAP, age-male-albumin-bilirubin-platelet; ALBI, albumin-bilirubin; AFP, alpha-fetoprotein; AST, aspartic transaminase; ALT, alanine transaminase; PLT, platelet; TBIL, total bilirubin; $\gamma$-GT, gamma-glutamyltransferase. 